\title{
ON SOME POLYNOMIAL VALUES OF REPDIGIT NUMBERS
}

\author{
T. KOVÁCS, GY. PÉTER, N. VARGA (DEBRECEN)
}

\begin{abstract}
We study the equal values of repdigit numbers and the $k$ dimensional polygonal numbers. We state some effective finiteness theorems, and for small parameter values we completely solve the corresponding equations.
\end{abstract}

Let

(1) $f_{k, m}(x)=\frac{x(x+1) \cdots(x+k-2)((m-2) x+k+2-m)}{k !}$

be the $m$ th order $k$ dimensional polygonal number, where $k \geq 2$ and $m \geq 3$ are fixed integers. As special cases for $f_{k, 3}$ we get the binomial coefficient $\left(\begin{array}{c}x+k-1 \\ k\end{array}\right)$, for $f_{2, m}(x)$ and $f_{3, m}(x)$ we have the corresponding polygonal and pyramidal numbers, respectively. These figurate numbers have already been investigated from several aspects and therefore have a rich literature, see Dickson [9]. For example, the question whether a perfect square is a binomial coefficient, i.e., if $f_{k, 3}(x)=f_{2,4}(y)$ and also the more general question on the power values of binomial coefficients was resolved by Györy [12]. The equation $\left(\begin{array}{l}x \\ n\end{array}\right)=\left(\begin{array}{l}y \\ 2\end{array}\right)$ has been investigated by several authors, for general effective finiteness statements we refer to Kiss [17] and Brindza [6]. In the special cases $m=3,4,5$ and 6 , the corresponding diophantine equations were resolved by Avanesov [1], Pintér [19] and de Weger [23] (independently), Bugeaud, Mignotte, Stoll, Siksek, Tengely [8] and Hajdu, Pintér [13], respectively. The equal values of polygonal and pyramidal numbers were studied by Brindza, Pintér, Turjányi [7] and Pintér, Varga [20].

Another important class of combinatorial numbers is the numbers of the form $d \cdot \frac{10^{n}-1}{10-1}, 1 \leq d \leq 9$. They are called repdigits and for $d=1$, repunits. Various results and conjectures have been stated concerning prime repunits and certain diophantine problems related to repdigits, see [11] and Chapter 12 in [22], respectively. For example, Ballew

Date: February 28, 2012.

2000 Mathematics Subject Classification. 11Y50,14H52,11B83.

Key words and phrases. Polygonal numbers, repdigit numbers, elliptic equations. 
and Weger [4] proved earlier that there are only six numbers, namely $1,3,6,55,66,666$ that are both triangular and repdigit numbers. Recently, Jaroma [14] gave an elementary proof of the fact that 1 is the only triangular repunit number. Keith [16] investigated the problem to determine which polygonal numbers are repdigits and solved it for numbers less than $10^{7}$. He also introduced an efficient algorithm for finding repdigit polygonal numbers and gave a complete characterization of all such numbers up to 50 digits.

One can also define the so-called generalized repunits with the formula $\left(b^{n}-1\right) /(b-1)$ for an integer $b \geq 2$. Dubner [10] gave a table of generalized repunit primes and probable primes for $b$ up to 99 and for large values of $n$.

In this paper we study the equal values of repdigits and the $k$ dimensional polygonal numbers. We state some effective finiteness theorems, and for small parameter values we completely solve the corresponding equations.

\section{NEW RESULTS}

A common generalization of repdigits and generalized repunits are numbers of the form

$$
d \cdot \frac{b^{n}-1}{b-1}
$$

i.e., taking repdigits with repeating digit $d$ in the number system of base $b$, where $1 \leq d<b$ and $b \geq 2$ integers.

We consider equation

$$
d \cdot \frac{b^{n}-1}{b-1}=f_{k, m}(x)
$$

and its special cases

$$
d \cdot \frac{10^{n}-1}{10-1}=f_{k, m}(x)
$$

and

$$
\frac{b^{n}-1}{b-1}=f_{k, m}(x)
$$

In our first result we represent an effective finiteness statement concerning the most general equation (2).

Theorem 1.1. Suppose that $k \geq 3$ or $k=2$ and $m=4$ or $m>13$. Then equation (2) has only finitely many integer solutions in $x$ and $n$, further,

$$
\max (|x|, n)<c,
$$


where $c$ is an effectively computable constant depending on $k, m, b$ and $d$. For $k=2$ and $m \in\{3,5,6,7,8,9,10,11,12\}$ equation (2) has infinitely many solutions for infinitely many values of the parameters $b, d$.

In the following two theorems we consider the special cases of equation (2) with repdigits or generalized repunits.

Theorem 1.2. Equation (3) with $k \geq 2$ has only finitely many integer solutions $n, x$ except for the values $(d, m)=(3,8)$. In these cases the equation has infinitely many solutions that can be given explicitly.

Theorem 1.3. Equation (4) with $k \geq 2$ has only finitely many integer solutions $n, x$ except for the values $(b, m)=(4,8),(9,3),(9,6),(25,5)$. In these cases the equation has infinitely many solutions that can be given explicitly.

In our numerical investigations, we take those polynomials $f_{k, m}(x)$, where $k \in\{2,3,4\}$. For each of these cases we let $d \in\{1,2, \ldots, 9\}$ and $m \in\{3,4, \ldots, 20\}$ and solve completely the corresponding equation. To state our numerical results, we need the following concept. A solution to equation (3) is called trivial if it yields $0=0$ or $1=1$. This concept is needed because of the huge number of trivial solutions; on the other hand, such solutions of (3) can be listed easily for any fixed $k$.

Theorem 1.4. All nontrivial solutions of equation (3) in case of $k=$ 2,3, respectively, are exactly those contained in Tables 1 and 2 respectively. If $k=4$ equation (3) has only trivial solutions.

\begin{tabular}{|c|c|c||c|c|c|}
\hline$(d, m)$ & Solutions $(n, x)$ & $f_{k, m}(x)$ & $(d, m)$ & Solutions $(n, x)$ & $f_{k, m}(x)$ \\
\hline$(1,9)$ & $(3,6)$ & 111 & $(6,3)$ & $(1,3)$ & 6 \\
\hline$(1,11)$ & $(2,2)$ & 11 & $(6,3)$ & $(2,11)$ & 66 \\
\hline$(1,14)$ & $(2,-1)$ & 11 & $(6,3)$ & $(3,-37)$ & 666 \\
\hline$(2,5)$ & $(3,-12)$ & 222 & $(6,6)$ & $(1,2)$ & 6 \\
\hline$(3,3)$ & $(1,2)$ & 3 & $(6,6)$ & $(3,-18)$ & 666 \\
\hline$(3,11)$ & $(3,9)$ & 333 & $(6,9)$ & $(1,-1)$ & 6 \\
\hline$(4,4)$ & $(1,2)$ & 4 & $(6,17)$ & $(3,-9)$ & 666 \\
\hline$(4,7)$ & $(1,-1)$ & 4 & $(7,5)$ & $(1,-2)$ & 7 \\
\hline$(5,3)$ & $(2,-11)$ & 55 & $(8,16)$ & $(2,4)$ & 88 \\
\hline$(5,6)$ & $(2,-5)$ & 55 & $(8,8)$ & $(1,2)$ & 8 \\
\hline$(5,7)$ & $(2,5)$ & 55 & $(9,4)$ & $(1,3)$ & 9 \\
\hline
\end{tabular}

TABLE 1 . The case of $f_{2, m}(x)$ 


\begin{tabular}{|c|c|c|}
\hline$(d, m)$ & Solutions $(n, x)$ & $f_{k, m}(x)$ \\
\hline$(1,6)$ & $(2,3)$ & 11 \\
\hline$(1,10)$ & $(2,2)$ & 11 \\
\hline$(4,3)$ & $(1,2)$ & 4 \\
\hline$(5,4)$ & $(1,2)$ & 5 \\
\hline$(5,4)$ & $(2,5)$ & 55 \\
\hline$(6,5)$ & $(1,2)$ & 6 \\
\hline$(7,6)$ & $(1,2)$ & 7 \\
\hline$(8,7)$ & $(1,2)$ & 8 \\
\hline$(9,8)$ & $(1,2)$ & 9 \\
\hline
\end{tabular}

TABLE 2. The case of $f_{3, m}(x)$

Remark. We considered some other related equations, corresponding to larger values of the parameter $k$ of the polynomial $f_{k, m}(x)$, that lead to genus 2 equations. However, because of certain technical difficulties, we could not solve them by the Chabauty method.

\section{PROOFS}

Lemma 2.1. Let $f(X)$ be a polynomial with rational integer coefficients and with at least two distinct roots. Suppose $b \neq 0, m \geq 0, x$ and $y$ with $|y|>1$ are rational integers satisfying

$$
f(x)=b y^{m} .
$$

Then $m$ is bounded by a computable number depending only on $b$ and $f$.

Proof. This is the main result of [21].

Proof of Theorem 1.1. Equation (2) is equivalent to

(5) $k ! d b^{n}=(b-1) x(x+1) \cdots(x+(k-2))((m-2) x+k+2-m)+d k !$

Let us assume first that $k \geq 4$. Our aim is to show that the polynomial on the right-hand side of $(5)$ is never an almost perfect power. On supposing the contrary we have

(6) $(b-1) x(x+1) \cdots(x+(k-2))((m-2) x+k+2-m)+d k !=c(x-\alpha)^{k}$,

with $c, \alpha \in \mathbb{Q}$. Substituting $x=0,-1,-2$ in equation (6), we obtain the equalities

$$
d k !=c(-\alpha)^{k}
$$

$$
d k !=c(-1-\alpha)^{k}
$$




$$
d k !=c(-2-\alpha)^{k}
$$

From (7) and (8) we get that

$$
c(-\alpha)^{k}=c(-1-\alpha)^{k},
$$

which yields that

$$
\left(\frac{1+\alpha}{\alpha}\right)^{k}=1
$$

Therefore $(1+\alpha) / \alpha$ is a rational root of unity, i.e., \pm 1 which means that $\alpha=-1 / 2$. On the other hand, considering (7) and (9), we obtain that

$$
c(-\alpha)^{k}=c(-2-\alpha)^{k} .
$$

Following a similar calculation we get that $\alpha=-1$, which is a contradiction. Therefore, our theorem follows from Lemma 2.1 for the case $k \geq 4$.

Now, let $k=3$. Then equation (5) has the form

$$
6 d b^{n}=(b-1) x(x+1)((m-2) x+5-m)+6 d .
$$

After carrying out the multiplications on the right-hand side we obtain that

$$
6 d b^{n}=(b-1)(m-2) x^{3}+3(b-1) x^{2}+(b-1)(5-m) x+6 d .
$$

Let us again assume that the right-hand side is an almost perfect power, i.e., equals $c(x-\alpha)^{3}$, with $c, \alpha \in \mathbb{Q}$. Then the original coefficients have the form

$$
\begin{gathered}
(b-1)(m-2)=c, \quad 3(b-1)=-3 c \alpha \\
(b-1)(5-m)=3 c \alpha^{2}, \quad 6 d=-c \alpha^{3} .
\end{gathered}
$$

From the first and second equation we get that $\alpha=\frac{1}{2-m}$. At the same time from the second and third equation we get that $\alpha=\frac{m-5}{3}$. This yields that $m \in \mathbb{C} \backslash \mathbb{R}$. Hence we derived a contradiction again. As in the previous case, Lemma 2.1 completes the proof for $k=3$.

In the remaining case let $k=2$. Then equation (5) has the form

$$
2 d b^{n}=(b-1) x((m-2) x+4-m)+2 d .
$$

If the right-hand side of (11) is an almost perfect square then

$$
(b-1)(m-2) x^{2}+(b-1)(4-m) x+2 d=c x^{2}-2 c x \alpha+c \alpha^{2}
$$

with rational $c$ and $\alpha$, further, on comparing the corresponding coefficients we have

$$
(b-1)(m-2)=c, \quad(b-1)(4-m)=-2 c \alpha, \quad 2 d=c \alpha^{2} .
$$


Hence we get that $\alpha=\frac{4-m}{4-2 m}$ and so $\frac{b-1}{d}=\frac{8(m-2)}{(4-m)^{2}} \geq 1$. This yields that $3 \leq m \leq 13$ integer and $m \neq 4$ and

$$
\frac{b-1}{d} \in\left\{\frac{88}{81}, \frac{5}{4}, \frac{72}{49}, \frac{16}{9}, \frac{56}{25}, 3, \frac{40}{9}, 8,24\right\} .
$$

This is satisfied by infinitely many pairs $b, d$. Therefore for infinitely many parameter values $b, d$ the right-hand side of equation (11) can be an almost perfect square which yields infinitely many integer solutions $n, x$ of equation (2). Otherwise, Lemma 2.1 gives our statement for $k=2$ and $m=4$ or $m>13$.

Proof of Theorem 1.2. For $k \geq 3$ the statement follows from Theorem 1.1. Now, let $k=2$. By a similar argument as in the proof of Theorem 1.1 , case $k=2$, we obtain that

$$
\frac{9}{d}=\frac{8(m-2)}{(4-m)^{2}}>0 .
$$

Since $d$ and $m$ are integers, their only possible value is $(d, m)=(3,8)$. Apart from this case the right-hand side of (11) cannot be a perfect square. Hence by Lemma 2.1 the theorem follows for $k=2$. In addition, in the exceptional case we show that equation (5) has infinitely many integer solutions $n, x$. Our equation is

$$
6 \cdot 10^{n}=54 x^{2}-36 x+6=54\left(x-\frac{1}{3}\right)^{2} .
$$

This yields that for arbitrary $k \in \mathbb{N}$ we have a solution $n=2 k$ and $x=-\frac{10^{k}-1}{3}$.

Proof of Theorem 1.3. For $k \geq 3$ the statement follows from Theorem 1.1. In case of $k=2$ a similar calculation has to be carried out as in the proof of Theorem 1.2. This yields the exceptional cases: $(b, m)=(4,8),(9,3),(9,6),(25,5)$. Showing that for these parameters the original equation has infinitely many solutions can be done similarly as in the previous proof.

Proof of Theorem 1.4. Let $k=2$. Then $f_{2, m}(x)=\frac{(m-2) x^{2}+(4-m) x}{2}$. Since the right-hand side of equation (3) is of degree 2 by reducing the left-hand side to a polynomial of degree 3 we obtain an elliptic equation which can further be solved by the program package Magma [5]. We illustrate these computations by an example. Set $(d, m)=(3,11)$. Then equation (3) is

$$
3 \cdot \frac{10^{n}-1}{9}=\frac{9 x^{2}-7 x}{2} .
$$


The left-hand side of this equation can be reduced to polynomials of degree 3 by considering $n \bmod 3$. If $n \equiv i(\bmod 3),(i=0,1,2)$ then $10^{n}=10^{3 k+i}$ for some $k \in \mathbb{Z},(i=0,1,2)$. Then substituting $y=10^{k}$, we get the following three distinct equations:

$$
\begin{gathered}
2 y^{3}-2=27 x^{2}-21 x, \\
20 y^{3}-2=27 x^{2}-21 x, \\
200 y^{3}-2=27 x^{2}-21 x .
\end{gathered}
$$

Multiplying both hand sides of equations (13a),(13b),(13c) by 108 , 10800, 1080000, respectively, and introducing the new variables $X_{1}=$ $54 x, Y_{1}=6 y ; X_{2}=540 x, Y_{2}=60 y ; X_{3}=5400 x, Y_{3}=600 y$; respectively, we obtain

$$
\begin{gathered}
Y_{1}^{3}-216=X_{1}^{2}-42 X_{1}, \\
Y_{2}^{3}-21600=X_{2}^{2}-420 X_{2}, \\
Y_{3}^{3}-2160000=X_{3}^{2}-4200 X_{3} .
\end{gathered}
$$

With the procedure IntegralPoints of Magma one can compute the integer points of these curves, and then determine the solutions $n, x$ of equation (12). The solution is exactly the one listed in Table 1.

Now let $k=3$. Then $f_{3, m}(x)=\frac{(m-2) x^{3}+3 x^{2}+(5-m) x}{6}$. Since the righthand side of equation (3) is of degree 3 by reducing the left-hand side to a polynomial of degree 2 we obtain an elliptic equation again which can be solved by Magma. We illustrate these computations by an example. Set $(d, m)=(4,3)$. Then equation $(3)$ is

$$
4 \cdot \frac{10^{n}-1}{9}=\frac{x^{3}+3 x^{2}+2 x}{6} \text {. }
$$

The left-hand side of this equation can be reduced to polynomials of degree 2 by considering $n$ modulo 2 . If $n \equiv i(\bmod 2),(i=0,1)$ then $10^{n}=10^{2 k+i}$ for some $k \in \mathbb{Z},(i=0,1)$. Then substituting $y=10^{k}$, we get the following two distinct equations:

$$
\begin{gathered}
8 y^{2}-8=3 x^{3}+9 x^{2}+6 x, \\
80 y^{2}-8=3 x^{3}+9 x^{2}+6 x .
\end{gathered}
$$

Multiplying both hand sides of equations (16a),(16b) by 72, 72000, respectively, and introducing the new variables $X_{1}=6 x, Y_{1}=24 y$; $X_{2}=60 x, Y_{2}=2400 y$; respectively, we obtain

$$
\begin{aligned}
Y_{1}^{2}-576 & =X_{1}^{3}+18 X_{1}^{2}+72 X_{1}, \\
Y_{2}^{2}-576000 & =X_{2}^{3}+180 X_{2}^{2}+7200 X_{2} .
\end{aligned}
$$


With the procedure IntegralPoints of Magma one can compute the integer points of these curves, and then determine the solutions $n, x$ of equation (15). The solution is exactly the one listed in Table 2.

Finally, let $k=4$. Now

$$
f_{4, m}(x)=\frac{(m-2) x^{4}+2 m x^{3}+(14-m) x^{2}+(12-2 m) x}{24} .
$$

Since the right-hand side of equation (3) is of degree 4 by reducing the left-hand side to a polynomial of degree 2 we obtain a genus 1 equation which can be solved by Magma. We take only one example again. Set $(d, m)=(3,5)$. Then equation $(3)$ is

$$
3 \cdot \frac{10^{n}-1}{9}=\frac{3 x^{4}+10 x^{3}+9 x^{2}+2 x}{24} .
$$

The left-hand side of this equation can be reduced to polynomials of degree 2 by considering $n$ modulo 2 . If $n \equiv i(\bmod 2),(i=0,1)$ then $10^{n}=10^{2 k+i}$ for some $k \in \mathbb{Z},(i=0,1)$. Then substituting $y=10^{k}$, we get the following two distinct equations:

$$
\begin{gathered}
8 y^{2}-8=3 x^{4}+10 x^{3}+9 x^{2}+2 x, \\
80 y^{2}-8=3 x^{4}+10 x^{3}+9 x^{2}+2 x .
\end{gathered}
$$

Multiplying both hand sides of equations (19a),(19b) by 2, 5, respectively, and introducing the new variables $Y_{1}=4 y, Y_{2}=20 y$; respectively, we obtain

$$
\begin{gathered}
Y_{1}^{2}=6 x^{4}+20 x^{3}+18 x^{2}+4 x+16 \\
Y_{2}^{2}=15 x^{4}+50 x^{3}+45 x^{2}+10 x+40
\end{gathered}
$$

The procedure IntegralQuarticPoints of Magma can find the integral points of such hyperelliptic curves as (20a) and (20b) if the constant term on the right-hand side is a square or a point of the curve is given. It just happens in case of (20a). Therefore using the previously mentioned command we determine all integral points of (20a) and substituting backwards, we find just the trivial solution $n=x=0$ to the original equation (18). In case of (20b) we cannot use the previous procedure. However the algorithm called IntGenQuartPoints.m implemented in Magma by Kovács [18] can be applied to this curve. This algorithm needs neither of the previous conditions on the hyperelliptic curve. Having the integral points of (20b) and substituting backwards again, we find no solution to the original equation (18). 
Acknowledgements. Research is supported in part by the OTKA grant K75566, the TÁMOP 4.2.1./B-09/1/KONV-2010-0007, TÁMOP4.2.2/B-10/1-2010-0024 projects implemented through the New Hungary Development Plan co-financed by the European Social Fund, and the European Regional Development Fund.

\section{REFERENCES}

[1] E. T. Avanesov, Solution of a problem on figurate numbers, Acta Arith. 12 (1966/67), 409-420.

[2] A. Baker, Transcendental number theory. Cambridge Mathematical Library. Cambridge University Press, Cambridge, second edition, 1990.

[3] A. Baker and H. Davenport, The equations $3 x^{2}-2=y^{2}$ and $8 x^{2}-7=z^{2}$, Quart. J. Math. Oxford Ser., 20 (1969), 129-137.

[4] D.W. Ballew, R.C. Weger, Repdigit triangular numbers, J. Recreational Math. $8(1975 / 76), 96-98$.

[5] W. Bosma, J. Cannon, C. Playoust, The Magma algebra system. I. The user language., J. Symbolic Comput. 24 (1997), 235-265.

[6] B. Brindza, On a special superelliptic equation, Publ. Math. Debrecen, 39 (1991), 159-162.

[7] B. Brindza, Á. Pintér and S. Turjányi, On equal values of pyramidal and polynomial numbers, Indag. Math. (N.S.), 9 (1998), 183-185.

[8] Y. Bugeaud, M. Mignotte, M. Stoll, S. Siksek, Sz. Tengely, Integral Points on Hyperelliptic Curves, Algebra and Number Theory 2 (2008) 859-885.

[9] L. E. Dickson, History of the theory of numbers, Vol. II: Diophantine analysis, Chelsea Publishing Co., New York, 1966, XXV+803 pp.

[10] H. Dubner, Generalized repunit primes, Math. Comp., 61 (1993), 927-930.

[11] R.L. Francis, Mathematical haystacks: another look at repunit numbers, College Math. J. 19 (1988), 240-246.

[12] K. Györy, On the Diophantine equation $\left(\begin{array}{l}n \\ k\end{array}\right)=x^{l}$, Acta Arith., 80 (1997), 289-295.

[13] L. Hajdu, Á. Pintér, Combinatorial diophantine equations, Publ. Math., Debrecen 56 (2000), 391-403.

[14] J.H. Jaroma, Triangular repunit - there is but 1, Czechoslovak Math. Journal, 60 (135) (2010), 1075-1077.

[15] M. Keith, Repdigit polygonal numbers, J. Recreational Math. 12 (1979/80), $9-15$.

[16] M. Keith, On Repdigit Polygonal Numbers, Journal of Integer Sequences, 1 (1998), Article 98.1.6.

[17] P. Kiss, On the number of solutions of the Diophantine equation $\left(\begin{array}{l}x \\ p\end{array}\right)=\left(\begin{array}{l}y \\ 2\end{array}\right)$, Fibonacci Quart., 26 (1988), 127-130.

[18] T. Kovács, Combinatorial numbers in binary recurrences, Period. Math. Hungar. 58 (2009) 83-98.

[19] Á. Pintér, A note on the Diophantine equation $\left(\begin{array}{l}x \\ 4\end{array}\right)=\left(\begin{array}{l}y \\ 2\end{array}\right)$, Publ. Math. Debrecen, 47 (1995), 411-415.

[20] Á. Pintér and N. Varga, Resolution of a nontrivial Diophantine equation without reduction methods, Publ. Math. Debrecen, 79 (2011), 605-610. 
[21] A. Schinzel and R. Tijdeman, On the equation $y^{m}=P(x)$, Acta Arith., 31 (1976), 199-204.

[22] T. N. Shorey, R. Tijdeman, Exponential diophantine equations, Cambridge University Press, Cambridge, 1986, X+240. pp.

[23] B. M. M. de Weger, A binomial Diophantine equation, Quart. J. Math. Oxford Ser. (2) 47 (1996), 221-231.

T. Kovács, Gy. Péter, N. Varga

University of Debrecen, Institute of Mathematics

H-4010 Debrecen, P.O. Box 12.

HUNGARY

E-mail address: tkovacs@science.unideb.hu, gyongyver.peter@econ.unideb.hu, nvarga@science.unideb.hu 